\title{
A Study of the Coverage of Large-scale Sensor Networks
}

\author{
Benyuan Liu \\ Department of Computer Science \\ University of Massachusetts Lowell \\ Lowell, MA 01854 \\ Email: bliu@cs.uml.edu
}

\author{
Don Towsley \\ Department of Computer Science \\ University of Massachusetts Amherst \\ Amherst, MA 01002 \\ Email: towsley@cs.umass.edu
}

\begin{abstract}
We study the coverage properties of large-scale sensor networks. Three coverage measures are defined to characterize the fraction of the area covered by sensors (area coverage), the fraction of sensors that can be removed without reducing the covered area (node coverage), and the capability of the sensor network to detect objects moving in the network (detectability), respectively. We approach the coverage problem from a theoretical perspective and explore the fundamental limits of the coverage of a large-scale sensor network. We characterize the asymptotic behavior of the coverage measures for a variety of sensor network scenarios. We find that the coverage of a sensor network exhibits different behaviors for different network configuration and parameters. Based on the analytical characterizations of the network coverage, we further discuss the implications to network planning and protocol performance of sensor networks.
\end{abstract}

\section{INTRODUCTION}

Advances in micro-sensor and communication technologies have made it possible to manufacture small sensors with simple sensing, processing, and wireless communication capabilities in a cost-effective fashion [1], [2]. A sensor network can be formed by deploying specialized sensors in the region of interest to perform certain sensing and networking tasks. Applications of sensor networks include battlefield surveillance, and environment monitoring [3], [4]. Many of these applications involve large-scale sensor networks, where a large number of sensors are deployed in a vast geographical area.

It is important to understand and characterize the performance of large-scale sensor networks. This helps to better design and use sensor networks for different application scenarios. In this work, we study a fundamental performance measure of a sensor network, namely, its coverage. Coverage of a sensor network represents the quality of service (surveillance) that it can provide, for example, how well a region of interest is monitored by sensors, how effective a sensor network is in detecting intruding objects.

There are many different measures for the coverage of a sensor network. Given a sensor network, we would like to know the fraction of the area covered by sensors (defined later as area coverage), which is a direct measure of sensor network coverage. The characterization of area coverage is important for sensor network planning. It can be used to determine how small the footprint of each sensor can be or how densely sensors should be deployed to guarantee that a fraction of the region is covered. Power consumption is another important concern in sensor networks, since the power supply of a sensor is usually limited. Many schemes have been proposed to reduce power consumption by turning off redundant sensors while ensuring that a certain fraction of area be covered [5], [6]. To this end, we are interested in the fraction of the sensors that can be removed without reducing the covered area (node coverage), which represents the redundancy level of the sensor deployment. For intrusion detection applications, it is important to know the capability of a sensor network to detect intruding objects. An object is detected if it goes through a covered area. In this regard, we study the detectability of a sensor network, which is defined to be the probability that a sensor network is able to detect objects that move inside or through a region of interest.

While most previous work on sensor network coverage focuses on coverage-related protocol and algorithm design [5], [6], [7], [8], [9], the goal of our work is to characterize the asymptotic behavior of the coverage of large-scale sensor networks and explore the fundamental limits of the network coverage. As in most of the previous work, we will focus on the coverage properties of a sensor network and will not address the communication and networking aspects of the network.

We consider a variety of network scenarios. In terms of the network topology, we study large-scale sensor networks that lie within a two-dimensional plane and a two-dimensional strip. The placement of sensors can vary significantly in different applications. In a "structured" sensor network application (e.g. video surveillance system), sensors are placed at the exact specific locations. While in an "unstructured" sensor network application (e.g. battlefield surveillance), sensors may be randomly dropped. In this work, we focus on the latter case where sensors are randomly placed in a field. In terms of the sensing model, we consider a Boolean sensing model and a more general sensing model, both widely adopted in the literature. For each scenario, we characterize the area coverage, the node coverage, and the detectability of the network. We find that the coverage of a sensor network exhibits different behaviors for different network configuration and parameters. Based on the analytical characterizations, we further examine the implications to protocol design and performance of sensor 
networks.

For a large-scale randomly placed sensor network under the Boolean sensing model, the area coverage is obtained using results in stochastic geometry. This is further used to derive the required sensor density to achieve a target area coverage. The simulation results of the node coverage suggest that if sensors are not deployed at a very high density, the fraction of redundant nodes that can be turned off without reducing covered area is small. The analytical characterizations of the detectability are obtained for unknown sensor location scenario. In this case, the probability of an intruding object being detected is minimized when the object takes a straight path, and the probability approaches one as the distance goes to infinity. If sensor locations are known, for a sensor network within a two-dimensional strip, an object can almost surely find a path to cross without being detected. For a sensor network within a two-dimensional plane, the detectability of the network exhibits a phase transition behavior at a critical density. If the sensor density is below the critical density, an intruding object can almost surely find a path to cross the network without being detected. However, if the sensor density is above the critical density, no path exists such that an object can cross the network without being detected.

For large-scale sensor networks under the general sensing model, we obtain analytical results for the area coverage. For node coverage, we prove that no sensor can be turned off without reducing the covered region. The detectability of a sensor network under the general sensing model is more difficult to obtain than in the Boolean sensing model. Nevertheless, we show that, if sensor density is above some critical value, no path exists such that an object can cross the network without being detected. We also conjecture that if the sensor density is below some critical value, an object can almost surely find a path to cross the network without being detected.

The remainder of the paper is organized as follows. In Section II, we review some of the related work on the coverage of sensor networks. In Section III, we describe the network model and define three coverage measures of large-scale sensor networks. In Section IV, we present the results on the coverage of large-scale sensor networks under the Boolean sensing model. The coverage results under a general sensing model are presented in Section V. Finally, we summarize this paper in Section VI.

\section{RELATED WORK}

In the past few years, coverage has been an active research area in sensor networks. Many studies have focused on characterizing the area coverage and designing algorithms to achieve desired area coverage. In [10], the authors studied the coverage of a grid-based wireless sensor network. They derived the necessary and sufficient conditions on the sensing range and failure rate of sensors in order to ensure that the whole network is covered as well as connected. In [5], the authors proposed a coverage-preserving scheme to extend sensor network lifetime. Redundant sensors that are fully covered by other sensors are turned off to reduce power consumption, while the fraction of the area covered by sensors is preserved. In [6], a probing-based density control algorithm is proposed to extend sensor network lifetime. Again, the basic idea is to turn off redundant sensors to save energy. A sleeping sensor wakes up occasionally to probe its neighborhood and starts working if there are no other working sensors in its probing range. The desired redundancy of working sensors can be achieved by adjusting the probing range of sensors.

Another line of work on coverage studies the path exposure of moving objects in sensor networks, which is a quantitative measure of how well sensors can detect objects moving in the network. In [8], the authors proposed algorithms to find paths which are most or least likely to be detected by sensors in a sensor network. The authors further defined and studied the path exposure of a moving object in a sensor network [7], which is a quantitative measure of how well an object, moving on an arbitrary path, can be detected by the sensor network. An algorithm is developed to find minimum exposure paths in sensor networks, where the probability of a moving object being detected is minimized. Along this line, [9] investigates deployment strategies for sensor networks performing target detection. The goal of sensor deployment is to maximize the exposure of the least exposed path in the network.

Most of these previous work has focused on protocol and system design, and studied the performance of the proposed algorithms. In this paper, we approach the coverage problems from a different perspective by studying the fundamental properties and limitations of a sensor network's coverage. These properties and limitations are determined by the basic network parameters (e.g. deployment strategy, sensor density, etc), but they can fundamentally impact the protocol performance in a sensor network. For example, when the sensor density is above some threshold (percolation density), no algorithm can find a path such that an object can cross the network without being detected.

\section{LARGE-SCALE SENSOR NETWORK MODEL}

In this section, we first describe the location model and two sensing models of the large-scale sensor networks used in our study. We then define three different coverage measures, namely, area coverage, node coverage, and detectability. In the rest of this paper, we will study these coverage measures of large-scale sensor networks under the location and sensing models.

\section{A. Location model}

We consider a network of a large number of sensors placed in a vast two-dimensional geographical region. We assume that the locations of sensors are uniformly and independently distributed in the region. Such a random initial deployment is desirable in scenarios where priori knowledge of the field is not available. Also, the random deployment can be the direct result of certain deployment strategies. For example, sensors may be air-dropped or launched via artillery in battlefields or unfriendly environments. Under this assumption, the locations 
of sensors can be modeled by a stationary two-dimensional Poisson point process. Denote the density of the underlying Poisson point process as $\lambda$, which is measured by the number of sensors per unit area. The number of sensors located in a region $A, N(A)$, follows a Poisson distribution of parameter $\lambda\|A\|$, where $\|A\|$ represents the area of the region.

$$
P(N(A)=k)=\frac{e^{-\lambda\|A\|}(\lambda\|A\|)^{k}}{k !}
$$

\section{B. Sensing models}

In this work, we consider two different sensing models: a Boolean sensing model and a general sensing model that considers more realistic sensing characteristics of a sensor, as well as the possible collaborations among sensors. Both models have been widely used in the literature [5], [6], [7], [9], [10].

1) Boolean sensing model: In the Boolean sensing model, each sensor has a certain sensing range, $r$. A sensor can only sense the environment and detect events within its sensing range. The simple Boolean sensing model has been widely adopted in the study of sensor networks [5], [6], [10].

A location is said to be "covered" by a sensor if it lies within the sensor's sensing area. The space is partitioned into two regions, the covered region, which is the region covered by at least one sensor, and the vacant region, which is the complement of the covered region. An object is detected if it passes through the covered region. For the purpose of computing the detectability of a sensor network, we say two sensors at locations $X_{i}$ and $X_{j}$ are directly connected if the sensing areas of the two sensors intersect, or equivalently, $\left|X_{i}-X_{j}\right| \leq 2 r$, where $\left|X_{i}-X_{j}\right|$ is the distance between the two sensors. A cluster is defined to be a connected component of the covered region. Two nodes are said to be connected if they belong to the same cluster. An object cannot traverse through a cluster without being detected, since it will need to go through a covered region and thus be detected.

2) General sensing mode: Despite its simplicity, the above Boolean sensing model does not capture the degradation of a sensor's sensing capability as the distance between the sensor and measuring point increases. In general, sensors have widely different sensing characteristics. Depending on the specific sensor device and application environment, different sensing models can be constructed to capture the sensing characteristics of the sensors. In this work, we adopt the following general sensing model, which has been widely used in the literature [7], [9], [11], [12], [13], and is reasonable for radio, acoustic and seismic signals.

For a sensor $s$, the sensing signal at an arbitrary point $p$ is given by:

$$
S(s, p)= \begin{cases}\frac{\alpha}{d(s, p)^{\beta}} & A \leq d(s, p)<B \\ 0 & \text { otherwise }\end{cases}
$$

where $\alpha$ is the energy emitted by events occurring at point $p$; $d(s, p)$ is the Euclidean distance between sensor $s$ and point $p$; parameters $A$ and $B$ define the range of a sensor's sensing capability. The sensing signal decays according to a power law with exponent $\beta$. The value of the decaying exponent is assumed to be known (or estimated via experiments). For radio signal sensing, the exponent typically ranges from 2.0 to 5.0 [11].

As in [7], we define the all-sensor field intensity of an arbitrary point $p, I_{p}$, to be the sum of the sensing signals of all sensors $\left(s_{1}, s_{2}, \ldots\right)$ at $p$, i.e.,

$$
I_{p}=\sum_{i=1}^{\infty} S\left(s_{i}, p\right)=\sum_{i=1}^{\infty} \frac{\alpha}{d\left(s_{i}, p\right)^{\beta}}
$$

We say that a point $p$ is covered if the all-sensor field intensity at $p$ is greater than or equal to some threshold, $\theta$, i.e., $I_{p} \geq \theta$. The set of points that are covered according to the above definition is called the covered region. Similarly, the complement of the covered region is called the vacant region. Note that this definition of coverage embodies the notion of value fusion in sensor networks, where sensors collaborate to determine whether an event occurs at a location. This definition requires the information of all of the sensors in the network to compute the all-sensor field intensity. The requirement can be relaxed by considering only the contributions from the $N \geq 1$ closest sensors. We refer to $I_{p}$ in this case as the $N$-closest sensor field intensity and to $I_{p}$ given by (3) as the all-sensor field intensity. Since all-sensor field intensity is strictly larger than $N$-closest sensor field intensity for any finite $N$, the covered region under all-sensor field intensity is a super set of the covered region under $N$-closest sensor field intensity. The all-sensor field intensity scenario characterizes the best coverage that a sensor network can offer. Note that the Boolean sensing model can be easily derived from the $N=1$ case. In this paper, we will study the sensor network coverage under the Boolean sensing model $(N=1)$ and the all-sensor field general sensing model $(N \rightarrow \infty)$, each being extremes of a spectrum.

Although the obtained results in this work are for the specific sensing signal and all-sensor field intensity function defined above, the same or similar techniques may be applied to other sensing model and sensor field intensity functions.

\section{Coverage measures}

To characterize the coverage of a sensor network quantitatively, we define the following three measures of the coverage.

Definition 1: Area coverage $\left(f_{a}\right)$ : the fraction of the geographical area covered by one or more sensors.

Note that the definition of a location being covered depends on the specific sensing model under consideration. In the Boolean sensing model, a location is covered if it is within the sensing area of a sensor. In the general sensing model, a location is covered if the all-sensor field intensity is greater than the threshold $\theta$. The area coverage is a very direct measure of how well a sensor network covers a region.

Definition 2: Node coverage fraction $\left(f_{n}\right)$ : the fraction of sensors that can be removed without reducing the coverage area. 
The node coverage measure represents the redundancy level of sensor deployment and has a direct effect on the performance of energy-efficient protocols that turn off redundant sensors while preserving area coverage [5].

Definition 3: Detectability $\left(p_{d}(S, D)\right)$ : the probability that a sensor network can detect an object moving from point $S$ to point $D$ in the network.

This is a measure of the intrusion detection capability of a sensor network. Obviouly, detectability depends on the deployment of the sensors and the locations of the two end points. Here we study the detectability in an expected sense, i.e., the expected value of all different realizations of the network. Therefore, the detectability of a sensor network is only a function of the density of sensors and the distance between the two end points $(l=|S D|)$.

In some specific sensor network scenarios, we are interested in variations of the above definition of detectability. We will introduce these variations when we describe those specific network scenarios.

Detectability of a sensor network is of interest in an application scenario such as detecting moving targets in battlefield and wild life tracking in environment monitoring. Note that in the Boolean sensing model, an object is detected by a sensor if it enters the sensing area of that sensor; while in the general sensing model, an object is detected at a location if the allsensing field intensity of the object at the location is greater than a threshold $\theta$.

\section{RANDom Sensor Networks: Boolean SENSING MODEL}

In this section, we study the coverage of large-scale random sensor networks under the Boolean sensing model. We consider networks within a two-dimensional infinite plane and two-dimensional strip, and study the area coverage, node coverage, and detectability for each case.

\section{A. Two-dimensional plane case}

\section{1) Area coverage:}

Theorem 1: Consider a two-dimensional infinite plane where sensors of sensing range $r$ are deployed uniformly at density $\lambda$, the area coverage of such a sensor network is

$$
f_{a}=1-e^{-\lambda \pi r^{2}}
$$

Proof. This is a result in stochastic geometry [14]. Here we present the key arguments of the proof. Consider a model where the locations of the sensors are distributed following a two-dimensional Poisson point process $P \equiv\left\{\xi_{i}, i \geq 1\right\} \in$ $(-\infty, \infty)^{2}$. Each point $\xi_{i}$ has a random shape $S_{i}$ centered at the point. Let $\alpha=E\left\|S_{i}\right\|<\infty$ denote the expected area of the random shape. In this case, each sensor has a disk shape and $\alpha=\pi r^{2}$.

For an arbitrary point $p$ in a large region $A$, the number of sensors located in this region is a Poisson random variable $N$, and $E(N)=\lambda\|A\|$. The probability that point $p$ does not lie within an arbitrary sensor equals $1-\alpha /\|A\|$. Since the sensors are located uniformly in $A$, conditioned on the number

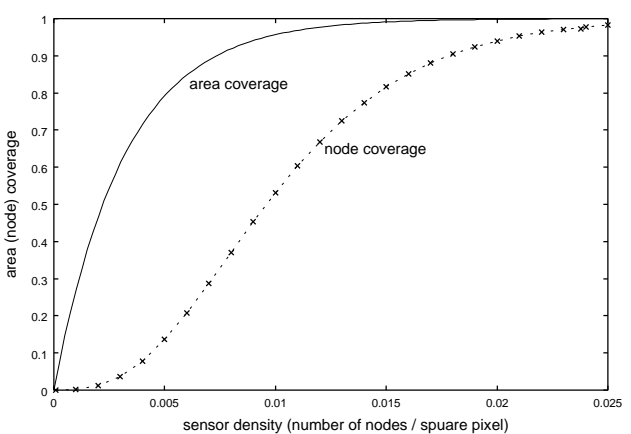

Fig. 1. Area and node coverage as a function of node density

of sensors $N$, the probability that point $p$ does not lie within any sensor equals $(1-\alpha /\|A\|)^{N}$. In the limit when $\|A\| \rightarrow \infty$, we have

$$
\begin{aligned}
P(\text { point } p \text { not covered }) & =E\left[\{1-\alpha /\|A\|\}^{N}\right] \\
& =e^{-(\alpha /\|A\|) E(N)} \\
& =e^{-\alpha \lambda} \\
& =e^{-\lambda \pi r^{2}}
\end{aligned}
$$

The fraction of the area being covered is simply

$$
f_{a}=1-P(\text { point } p \text { not covered })=1-e^{-\lambda \pi r^{2}}
$$

This formula can be used in network planning to determine the required sensor density in order to achieve a desired area coverage $f_{a}\left(0<f_{a}<1\right)$ almost surely. The density required is given by

$$
\lambda=-\ln \left(1-f_{a}\right) / \pi r^{2}
$$

2) Node coverage: It is difficult to obtain a closed form expression for node coverage in a two-dimensional sensor network. In Figure 1, we plot the simulation results of node coverage $\left(f_{n}\right)$ as a function of node density. The area coverage (equation (4)) is also plotted in Figure 1 to illustrate the relative difference between the two coverage measures. In the simulation, we approximated an infinite plane by a domain of 10000 x 10000 pixels. The density of the sensor nodes is measured in pixels ${ }^{-2}$. The sensing range of each sensor is set to be 10 pixels.

We observe that node coverage remains below $1 \%$ until the density of the sensor nodes increases to $2 \times 10^{-3}$. At this density, $47 \%$ of the area is covered by sensors. To provide a visual illustration of the area coverage and node coverage at this density, we show a small part of the network in Figure 2. This part is randomly chosen within the domain and contains a large number of sensors. It is representative of the whole network. In the illustration, each sensor is represented by a circle with a common sensing range, while filled circles represent those sensors that are fully covered by other sensors. We observe that the density is quite high and redundant sensors that are fully covered by other sensors are 


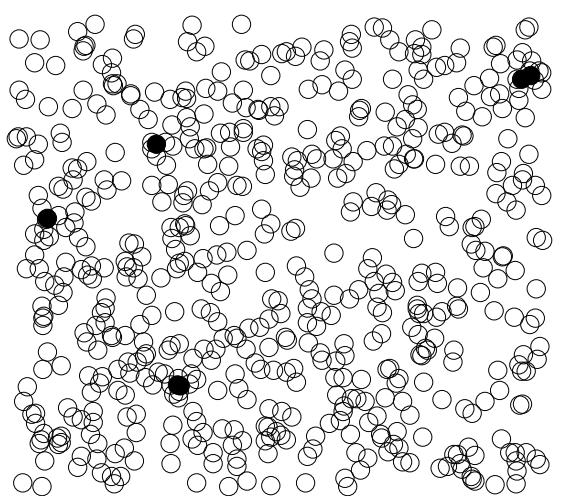

Fig. 2. Part of the sensor network at node density of $2 \times 10^{-3}$.

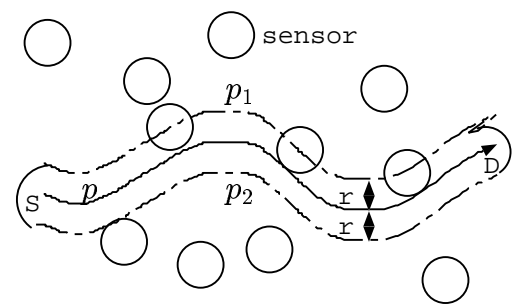

Fig. 3. Detecting zone of path $p$ between end points $S$ and $D$. The area of the detection zone is minimized when target takes a straight path between the two end points.

only sparsely distributed in the network. This result suggests that in scenarios where sensors cannot be deployed at a high density due to cost or other reasons, the fraction of redundant nodes that can be turned off without reducing area coverage is small.

3) Detectability: We study the detectability of a sensor network in two different scenarios. In the first scenario, we assume that the locations of sensors are unknown to intruding objects. In the second scenario, sensor locations are known to intruding objects.

(i) Unknown sensor locations.

Figure 3 depicts a scenario where an object moves from point $S$ to point $D$ along path $p$. We define the domain between two curves $\left(p_{1}\right.$ and $p_{2}$ ) of distance $r$ from $p$ as the detecting zone of path $p$ and denoted it as $Z_{p}$. An object moving along path $p$ will be detected by sensors located within $Z_{p}$. Denote the area of $Z_{p}$ as $\left\|Z_{p}\right\|$, the number of sensors in $Z_{p}, N_{p}$, is a Poisson random variable of parameter $\lambda\left\|Z_{p}\right\|$. Therefore, the probability an object moving along path $p$ being detected is

$$
P_{d}(p)=1-P\left(N_{p}=0\right)=1-e^{-\lambda\left\|Z_{p}\right\|}
$$

As can be directly derived from (6), in order to minimize the probability of being detected, an object should choose a path whose detecting zone area is minimized. For all possible paths between $S$ and $D$, the straight path yields the smallest detecting zone. Therefore, to minimize the probability of being detected, the best strategy is to take the direct path. Denote the length of segment $S D$ as $x$, we have $\left\|Z_{p}\right\|=2 r x+\pi r^{2}$. The probability of an object being detected along $S D$ is

$$
P_{d}(x)=1-e^{-\lambda\left(2 r x+\pi r^{2}\right)}
$$

As the distance between two end points approaches infinity $(x \rightarrow \infty)$, the detectability $\left(P_{d}^{\infty}\right)$ of a sensor network can be interpreted as the capability of the sensor network in detecting objects attempting to cross the network. We will call the detectability in this case, $P_{d}^{\infty}$, the asymptotic detectability of a sensor network. From equation (7), we have

$$
P_{d}^{\infty} \rightarrow 1, \text { as } x \rightarrow \infty
$$

Therefore, without knowing the locations of the sensors, an object cannot cross the network without being detected.

(ii) Known sensor locations.

Unfortunately, it is difficult to compute the detectability for the general case where the distance between the two end points is a parameter. Nevertheless, we have derived the asymptotic detectability as the distance between the two end points approaches infinity. Note that the asymptotic detectability represents a sensor network's capability to detect objects attempting to cross the network.

In the limit, as the distance between the two end points approaches infinity, the asymptotic detectability of a sensor network can be related to the continuum percolation of the sensors. According to percolation theory [15], there exists a critical density $\lambda_{c}$, where a phase transition occurs with respect to the size of the largest cluster.

If the density is below the critical density $\lambda_{c}$, all clusters are finite in size almost surely, and there is no percolation. In this case, there is a unique unbounded vacant component in the network. An object can cross the network without being detected by traversing through the unbounded vacant component. Otherwise, if there is no path that an object can cross the network without being detected, there must be an unbounded cluster percolating through the network, contradicting the fact that there is no percolation.

When the sensor density is above the critical density $\lambda_{c}$, an unbounded sensor cluster percolates the whole network almost surely, which will detect any object trying to cross the network. Therefore,

$$
P_{d}^{\infty}= \begin{cases}0 \text { a.s. } & \text { if } \lambda<\lambda_{c} \\ 1 \text { a.s. } & \text { if } \lambda \geq \lambda_{c}\end{cases}
$$

Note that because of the "zero-one" law of the phase transition, the asymptotic detectability cannot be anything other than 0 or 1 . Therefore, in sensor network planning, in order to provide effective intrusion detection for the worstcase scenario, sensors need to deployed at a density above the critical density. The critical density $\left(\lambda_{c}\right)$ depends on the sensing range of the sensors and can be obtained via simulation. In the above simulation setting, $\lambda_{c} \simeq 3.53 \times 10^{-3}$. Under this critical density, the area coverage $f_{a}$ is approximately 0.67 . Note that percolation theory has been used in [16] to study the connectivity of large-scale ad hoc networks, the detectability problem in our case is essentially a dual problem of the connectivity problem in [16]. 


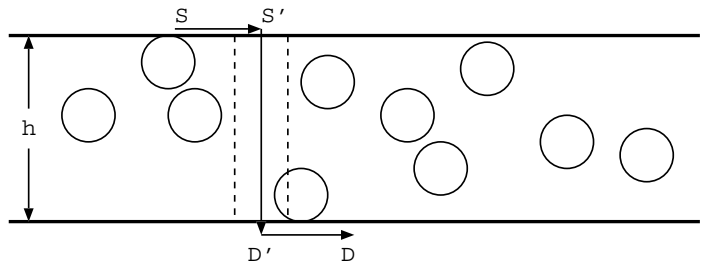

Fig. 4. Detectability of two-dimensional strip sensor network

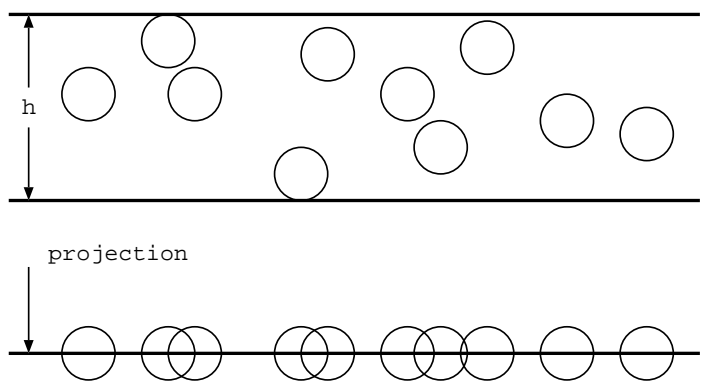

Fig. 5. Two-dimensional strip case

\section{B. Two-dimensional strip case}

In some scenarios, due to geographical terrain constraints or application requirements, the region of interest may have a long extension in one particular direction. The sensor network can be better modeled by a two-dimensional strip in this case, as shown in Figure 4.

For convenience, we set the boundaries of a twodimensional strip tangent to the sensing areas of most outlying sensors at the top and at the bottom. If the width of a twodimensional strip is much larger than the sensing range of the sensors, i.e., $h \gg r$, the boundary effect to area coverage and node coverage is small. In this case, the area coverage can be approximated by Equation (4); and the node coverage still remains small until the node density is sufficiently high, as in the two-dimensional infinite plane case.

In this scenario, it is more interesting to study the detectability where the two end points are on opposite sides of the strip. This corresponds to the capability of a sensor network to detect objects attempting to cross the strip from top to bottom.

(i) Unknown sensor locations.

It is easy to show that an object should cross the strip perpendicularly at some arbitrary point in order to minimize the chance of being detected, as illustrated in Figure 4. Based on (7), neglecting the boundary effect, the probability of an object being detected is

$$
P_{d}(2 \text {-dim strip })=1-e^{-2 \lambda r h}
$$

(ii) Known sensor locations.

For the two-dimensional infinite plane case, we have shown that there is a phase transition in the detectability of the sensor network that is due to the percolation of sensors in the network at a critical density. Above the critical density, an infinite cluster of sensors percolates the region, and no object can pass through without being detected.

However, for a strip of finite width $h$, percolation never occurs. This has been shown in [16] in the context of network connectivity. Projecting the two-dimensional strip on horizontal axis, one obtains a one-dimensional Poisson point process of density $\lambda h$, as shown in Figure 5. Since the distance between two nodes in the projected one-dimensional space is smaller than the original distance in two-dimensional space, existing connections between sensors are preserved while new connections are created. It has been shown in [17] that percolation never occurs in the one-dimensional case, which implies percolation never occurs on the two-dimensional strip. Therefore, there is almost surely a path in the two-dimensional strip where an object can cross without being detected by sensors.

$$
P_{d}(2 \text {-dim strip })=0
$$

The above coverage results are obtained for twodimensional sensor networks. Note that the same techniques can be used to derive coverage measures for large-scale threedimensional random sensor networks. Although the exact forms of the results may differ, the qualitative behavior of the coverage measures should be similar.

\section{RANDOM SENSOR NETWORK: GENERAL SENSING MODEL}

In this section, we study the area coverage, node coverage, and detectability for large-scale sensor networks under the general sensing model.

\section{A. Area coverage}

For an arbitrary point $p$, the all-sensor intensity function is $I_{p}=\sum_{i=1}^{\infty} \frac{\alpha}{d\left(s_{i}, p\right)^{\beta}}$. The necessary and sufficient condition for the convergence of $I_{p}$ is given in [18]:

$$
\int_{y}^{\infty} \frac{\alpha}{t^{\beta-1}} d t<\infty \text {, for a sufficiently large } y
$$

In this work, we assume that $\beta>2$ [11]. Thus, the allsensor field intensity $I_{p}$ converges for an arbitrary point $p$ in a network almost surely.

To derive the area coverage, we first obtain the probability density function (PDF) of the all-sensor field intensity of an arbitrary point $p, f_{I_{p}}(x)$. The homogeneity of the Poisson point process implies the probability density function is the same for all points in the plane.

As defined in Equation (3), $I_{p}=\sum_{i=1}^{\infty} \frac{\alpha}{d\left(s_{i}, p\right)^{\beta}}$, the allsensor field intensity is the sum of the sensing signals of all sensors in a network. Since the locations of sensors form a two-dimensional Poisson point process, the all-sensor field intensity of points in a sensor network can be modeled by a two-dimensional Poisson shot noise process [19], [20], [18], where the shot noise is synonymous here with the sensing signal function, defined in Equation (2). 


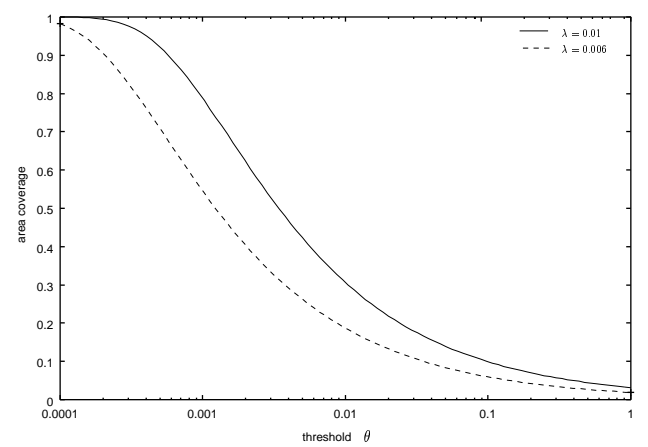

Fig. 6. Area coverage under general sensing model when $\alpha=1, \beta=4$

The probability density function of a one-dimensional Poisson shot noise model has been studied in [19], [20]. In this work, we derived the PDF of $I_{p}$ for a two-dimensional Poisson shot noise model. The derivation is quite involved and is presented in a more detailed report[17]. Denote $D=\frac{2}{\beta}$, we have

$$
f_{I_{p}}(x)=\frac{1}{2 \pi} \int_{-\infty}^{+\infty} \exp \left[-j \omega x-\lambda \pi \alpha^{D}(-j \omega)^{D} \Gamma(1-D)\right] d \omega
$$

Similar to [19], we can write the infinite sum form of the probability density function as

$f_{I_{p}}(x)=\frac{1}{\pi x} \sum_{n=1}^{\infty} \frac{(-1)^{n+1} \Gamma(1+n D) \sin (\pi n D)}{n !}\left[\frac{\lambda \pi \alpha^{D} \Gamma(1-D)}{x^{D}}\right]^{n}$

where the Gamma function $\Gamma(x)$ is defined as $\int_{0}^{\infty} t^{x-1} e^{-t} d t$.

Recall that a point is covered if $I_{p} \geq \theta$. Once we have the probability density function of the all-sensor field intensity, the area coverage can be computed as follow:

$$
f_{a}=P(\text { point } p \text { is covered })=P\left(I_{p} \geq \theta\right)=\int_{\theta}^{\infty} f_{I_{p}}(x) d x
$$

In general, the probability density function of $I_{p}$ and hence the area coverage can be computed numerically [19], [21]. As in [19], for the special case $D=1 / 2$, or $\beta=4$, we obtain the following closed-form result.

$$
f_{I_{p}}(x)=\frac{\lambda \pi \alpha^{1 / 2}}{2} x^{-3 / 2} \exp \left[-\frac{\lambda^{2} \pi^{3} \alpha}{4 x}\right]
$$

Therefore, the area coverage is

$$
f_{a}=\int_{\theta}^{\infty} f_{I_{p}}(x) d x=1-\frac{1}{\sqrt{\pi}} \Gamma\left(\frac{1}{2}, \frac{\alpha \lambda^{2} \pi^{3}}{4 \theta}\right)
$$

Figure 6 shows the area coverage for the above special case of $\beta=4$. We set $\alpha=1$ and consider two different sensor densities, $\lambda=0.006,0.01$. As can be observed from the figure, for the same threshold value, as the sensor density increases, the all-sensor field intensity of a location increases and the location becomes more likely to be covered. As a result, the area coverage increases. For the same sensor density value, as the threshold increases, a location becomes less likely to be covered. The area coverage is thus reduced.

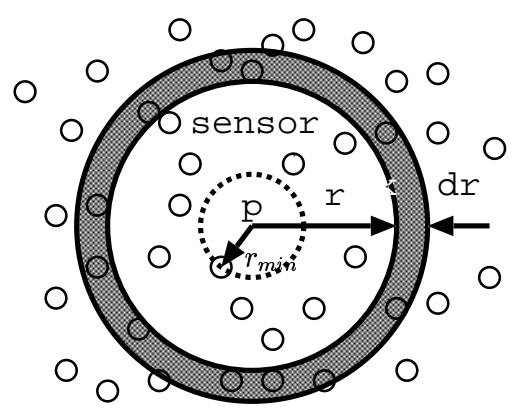

Fig. 7. Approximation of all-sensor fi eld intensity

\section{B. Node Coverage}

Theorem 2: In the general sensing model, no sensor can be turned off without reducing the covered region, or $f_{n}=0$.

Proof. We first show that there exist points in the network that are not covered. Consider an arbitrary point $p$ in the network. The distance between $p$ and the nearest sensor, $R_{\min }$, is a random variable whose distribution density function (PDF) is given by

$$
f_{R_{\min }}(x)=\lambda 2 \pi x e^{-\lambda \pi r^{2}}
$$

Denote $r_{c}=\left[\frac{2 \pi \lambda \alpha}{(\beta-2) \theta}\right]^{\frac{1}{\beta-2}}$, given $R_{\min }=r_{c}$, the expected all-sensor field intensity of point $p$ can be approximated as follows. Consider an infinitesimal ring structure of width $d r$ which is a distance $r$ away from point $p$ (see Figure 7), the area of the ring is $2 \pi r d r$. Hence, the number of sensors located within the ring is $\lambda 2 \pi r d r$, and each of these sensors contributes $\frac{\alpha}{r^{\beta}}$ to the all-sensor field intensity. Therefore, the all-sensor field intensity can be obtained by integrating the contributions of the ring structure from minimum radius $r_{c}$ to infinity. Therefore,

$$
E\left[I_{p} \mid R_{\text {min }}=r_{c}\right]=\int_{r_{c}}^{\infty} \lambda 2 \pi r \frac{\alpha}{r^{\beta}} d r=\frac{2 \pi \lambda \alpha}{\beta-2} \frac{1}{\left(r_{c}\right)^{\beta-2}}=\theta
$$

From the above derivation, we see that $E\left[I_{p} \mid R_{\min }=r_{c}\right]$ is a decreasing function of the minimum distance $r$. We have

$$
E\left[I_{p} \mid R_{\min }>r_{c}\right]<\theta
$$

Therefore,

$$
P\left(I_{p} \geq \theta \mid R_{\min }>r_{c}\right)<1
$$

or equivalently,

$$
P\left(I_{p}<\theta \mid R_{\min }>r_{c}\right)>0
$$

Since there is a non-zero probability for $R_{\min }>r_{c}$, i.e., $P\left(R_{\min }>r_{c}\right)>0$, there exist uncovered points $\left(I_{p}<\theta\right)$ in the network.

For the all-sensor intensity function of an arbitrary point $p$, we order the index of sensors according to their distances to $p$, such that $\forall i<j, d\left(s_{i}, p\right) \leq d\left(s_{j}, p\right)$. Define $I_{p}(n)=$ $\sum_{i=1}^{n} \frac{\alpha}{d\left(s_{i}, p\right)^{\beta}}$, we have $I_{p}=\lim _{n \rightarrow \infty} I_{p}(n)$, which converges 


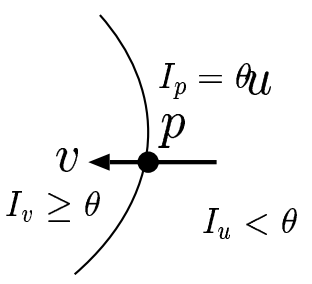

Fig. 8. Existence of point $p$ such that $I_{p}=\theta$

under our assumption $\beta>2$. Denote the coordinates of sensor $s_{i}$ as $\left(x_{i}, y_{i}\right)$ and the coordinates of $p$ as $(x, y)$, since each term $\frac{\alpha}{d\left(s_{i}, p\right)^{\beta}}=\frac{\alpha}{\left(\left(x_{i}-x\right)^{2}+\left(y_{i}-y\right)^{2}\right)^{\beta / 2}}$ is a continous function of variables $x$ and $y, I_{p}$ is also a continous function of the location of $p$.

Above we have proved there exist uncovered points in the network. Consider an uncovered point $u$ and a covered point $v$ in the network, as shown in Figure 8 , we have $I_{u}<\theta$ and $I_{v} \geq \theta$ by definition. Since $I_{p}$ is a continuous function along the segment from $u$ to $v,(u, v]$, there exists a point $p \in(u, v]$ such that $I_{p}=\theta$.

Since $I_{p}=\sum_{i=1}^{\infty} \frac{\alpha}{d\left(s_{i}, p\right)^{\beta}}$, if any sensor is turned off, a nonnegative term will be missing in the summation, and $I_{p}$ will decrease below $\theta$. Therefore, no sensor can be turned off without reducing the covered region.

\section{Detectability}

Deriving the detectability under the general sensing model $\left(P_{d}^{G S M}\right)$ is more difficult than in the Boolean sensing model. Under the Boolean sensing model, the covered region is directly defined by the union of the disks (sensing areas) of the underlying Poisson point process. As a result, the detectability simply depends on the distribution of the number of sensors located in the detecting zone; and the asymptotic detectability is directly related to the percolation of the underlying Poisson Boolean model. However, under the general sensing model, the covered region is a two-dimensional random field which has a more complicated dependence on the underlying point process. This makes the detectability under the general sensing model more difficult to compute than in the Boolean sensing model. Nevertheless, we can obtain some results for the asymptotic detectability. In the following, we prove an important property of the asymptotic detectability under the general sensing model.

Theorem 3: There exists a critical density $\lambda_{c}$, such that the asymptotic detectability is one almost surely if the sensor density is above the critical density.

$$
P_{d}^{G S M}=1 \text { a.s., if } \lambda>\lambda_{c}
$$

Proof: The flow of the proof is as follows. For a sensor network under the general sensing model, we first construct a Poisson Boolean model, whose covered region is a subset of the original covered region under the general sensing model. Therefore, if no path exists such that an object can cross the network without being detected in the constructed Poisson Boolean model, there is no such path under the general sensing model either.

For a sensor network under the general sensing model, we construct the following Poisson Boolean model $B\left(\lambda, r_{c}\right)$, where the underlying point process is preserved, and $r_{c}=(\alpha / \theta)^{1 / \beta}$. We can show that a point in the covered region of the Poisson Boolean model is also in the covered region under the general sensing model, which implies the covered region of the Poisson Boolean model is a sub-set of the covered region under the general sensing model.

For a point $p$ in the covered region of the Poisson Boolean model, the distance between $p$ and its nearest sensor $s_{p}$ is smaller than or equal to $r_{c}$, i.e., $d\left(s_{p}, p\right) \leq r_{c}$. The all-sensor field intensity is strictly larger than the contribution from the nearest sensor. Hence,

$$
I_{p}=\sum_{i=1}^{\infty} \frac{\alpha}{d\left(s_{i}, p\right)^{\beta}}>\frac{\alpha}{d\left(s_{p}, p\right)^{\beta}} \geq \frac{\alpha}{r_{c}^{\beta}}=\theta
$$

For the Poisson Boolean model $B\left(\lambda, r_{c}\right)$, based on Equation (9), there exists a critical density $\lambda_{c}$. If the sensor density is larger than the critical density, an unbounded cluster forms and the asymptotic detectability is one almost surely. Since the covered region under the general sensing model is a superset of the covered region of the constructed Boolean model, nonexistence of a path along which an object can cross the network without being detected in the Boolean model implies non-existence of such a path in the general sensing model. Therefore, we have

$$
P_{d}^{G S M}=1 \text { a.s., if } \lambda>\lambda_{c}
$$

In the Boolean sensing model, we have shown that when the sensor density is below some critical density, the asymptotic detectability is almost surely zero. Under the general sensing model, we conjecture that there is a similar result: if the sensor density is below some critical density, the asymptotic detectability is zero almost surely.

\section{CONCLUSIONS}

In this paper, we studied three fundamental coverage measures of large-scale sensor networks: area coverage, node coverage, and detectability. These measures are determined by basic network parameters and have important implications on network planning and protocol performance of sensor networks. We consider a Boolean sensing model and a general sensing model, both widely adopted in the literature. For each sensing model, we study the coverage of network scenarios that can be modeled by a two-dimensional infinite plane and a two-dimensional strip case. 
For a large-scale random sensor network under the Boolean sensing model, the area coverage can be obtained using volume fraction results established in stochastic geometry. The simulation results of the node coverage suggest that if sensors are not deployed at a very high density, the fraction of redundant nodes that can be turned off without reducing covered area is small. We have obtained analytical characterizations of the detectability of a sensor network if targets do not know the locations of sensors. If sensor locations are known, for the two-dimensional strip case, an object can almost surely find a path to cross the strip without being detected. For the two-dimensional infinite plane case, the detectability of a sensor network exhibits a phase transition behavior at a critical density. If the sensor density is below the critical density, an intruding object can almost surely find a path to cross the network without being detected; however, if the sensor density is above the critical density, no path exists such that an object can cross the network without being detected.

For large-scale sensor networks under the general sensing model, we have obtained analytical results for the area coverage by modeling the all-sensor field intensity as a twodimensional Poisson shot noise process. For node coverage, we have proved that no sensor can be turned off without reducing the covered region. The detectability of a sensor network under the general sensing model is more difficult to obtain than in the Boolean sensing model. We have shown that, if sensor density is above some critical value, no path exists such that an object can cross the network without being detected. We conjecture that if the sensor density is below some critical value, an object can almost surely find a path to cross the network without being detected.

\section{REFERENCES}

[1] G. Pottie and W. Kaiser, "Wireless integrated network sensors," Communications of the ACM, vol. 43, May 2000.

[2] J. Kahn, R. Katz, and K. Pister, 'Mobile networking for smart dust," in Mobile Computing and Networking, 1999.

[3] A. Mainwaring, J. Polastre, R. Szewczyk, D. Culler, and J. Anderson, "Wireless sensor networks for habitat monitoring," in First ACM International Workshop on Wireless Sensor Networks and Applications, 2002.

[4] A. Baptista, T. Leen, Y. Zhang, A. Chawla, D. Maier, W. chang Feng, W. chi Feng, J. Walpole, and C. S. andJuliana Freire, 'Environmental observation and forecasting systems: Vision, challenges and successes of a prototype," in Proceedings of the International Society for Environmental Information Sciences Annual Conference (ISEIS'2003) on Systems Science and Information Technology for Environmental Applications, 2003.

[5] D. Tian and N. D. Georganas, "A coverage-preserving node scheduling scheme for large wireless sensor networks," in First ACM International Workshop on Wireless Sensor Networks and Applications, 2002, pp. 32 41.

[6] F. Ye, G. Zhong, S. Lu, and L. Zhang, 'Peas: A robust energy conserving protocol for long-lived sensor networks," in Proc. ICDCS, 2003.

[7] S. Meguerdichian, F. Koushanfar, G. Qu, and M. Potkonjak, "Exposure in wireless ad-hoc sensor networks," in Mobile Computing and Networking, 2001, pp. 139-150.

[8] S. Meguerdichian, F. Koushanfar, M. Potkonjak, and M. B. Srivastava, "Coverage problems in wireless ad-hoc sensor networks," in Proc. IEEE Infocom, 2001, pp. 1380-1387.

[9] T. Clouqueur, V. Phipatanasuphorn, P. Ramanathan, and K. K. Saluja, 'Sensor deployment stategy for target detection," in First ACM International Workshop on Wireless Sensor Networks and Applications, 2002.
[10] S. Shakkottai, R. Srikant, and N. Shroff, 'Unreliable sensor grids: Coverage, connectivity and diameter," in Proc. IEEE Infocom, 2003.

[11] M. Hata, "Empirical formula for propagation loss in land mobile radio services," IEEE Transactions on Vehicular Technology, vol. 29, pp. 317 325, Aug. 1980.

[12] D. Li, K. Wong, Y. Hu, and A. Sayeed, 'Detection, classifi cation, tracking of targets in micro-sensor networks," in IEEE Signal Processing Magazine, 2002.

[13] M. Chu, H. Haussecker, and F. Zhao, "Scalable information-driven sensor querying and routing for ad hoc heterogeneous sensor networks," Int'l J. High Performance Computing Applications, vol. 16, no. 3, 2002.

[14] P. Hall, Introduction to the Theory of Coverage Processes. John Wiley \& Sons, 1988.

[15] R. Meester and R. Roy, Continuum percolation. Cambridge University Press, 1996.

[16] O. Dousse, P. Thiran, and M. Hasler, 'Connectivity in ad-hoc and hybrid networks," in Proc. IEEE Infocom, 2002.

[17] B. Liu, 'Design and performance modeling of wireless networks," Ph.D. dissertation, University of Massachusetts, Amherst, 2003.

[18] D. Daley, "The defi nition of a multi-dimensional generalization of shot noise," J. Appl. Prob., vol. 8, pp. 128-135, Mar 1971.

[19] S. B. Lowen and M. C. Teich, 'Power-law shot noise," IEEE Trans. Inform. Theory, vol. 36, no. 6, pp. 1302-1318, Nov 1990.

[20] S. O. Rice, "Mathematical analysis of random noise," Bell Syst. Tech. Journal, vol. 23, pp. 282-332, July 1944.

[21] W. Press, B. Flannery, S. Teukolsky, and W. Vetterling, Numerical Recipes. Cambridge University Press, 1986. 